\title{
Comparison of Two ANN Methods for Classification of Spirometer Data
}

\author{
Sujatha C. Manoharan ${ }^{1}$, Mahesh Veezhinathan and Swaminathan Ramakrishnan ${ }^{2}$ \\ ${ }^{1}$ College of Engineering, Guindy, Anna University, Chennai - 600025. \\ ${ }^{2}$ Madras Institute of Technology Campus, Anna University, Chennai - 600044.
}

\begin{abstract}
In this work, classification of spirometric pulmonary function test data performed using two artificial neural network methods is compared and reported. The pulmonary function data $(\mathrm{N}=150)$ were obtained from volunteers, using commercially available Spirometer, and recorded by standard data acquisition protocol. The data were then used to train $(\mathrm{N}=100)$ as well as to test $(\mathrm{N}=50)$ the neural networks. The classification was carried out using back propagation and radial basis function neural networks. The results confirm that the artificial neural network methods are useful for the classification of spirometric pulmonary function data. Further, it appears that the Radial basis function neural network is more sensitive when compared to back propagation neural networks. In this paper, the methodology, data collection procedure and neural network based analysis are described in details.
\end{abstract}

Keywords: Spirometer, pulmonary function, obstructive disease, restrictive disease, back propagation neural network, radial basis function neural network.

\section{INTRODUCTION}

$\mathrm{R}$ ESPIRATORY DISEASES are curable by early detection. Respiratory function is commonly assessed by standard spirometric pulmonary function test [1]. Pulmonary function test can detect the presence and degree of pulmonary functional abnormalities. It can differentiate between obstructive, restrictive and mixed obstructive/restrictive pathology. It can help in the evaluation of the presence and degree of increased airway responsiveness, and assess the risk of therapeutic or diagnostic interventions. The test monitors the effects of therapy and contributes to an accurate prognosis of disease and disability [1], [2].

Obstructive and restrictive diseases are two main types of lung disease found with lung function tests. In obstructive lung conditions, the airways are narrowed, usually causing an increase in the time it takes to empty the lungs. Obstructive lung disease can be caused by conditions such as emphysema, bronchitis, infection (which produces inflammation), and asthma. In restrictive lung conditions, there is a loss of lung tissue, a decrease in the lung's ability to expand, or a decrease in the lung's ability to transfer $\mathrm{O}_{2}$ into the blood or $\mathrm{CO}_{2}$ out of the blood [2], [3]. Restrictive lung disease can be caused by conditions such as pneumonia, lung cancer, scleroderma, pulmonary fibrosis, sarcoidosis, or multiple sclerosis. Other restrictive conditions include some chest injuries, being very overweight (obesity), pregnancy, and loss of lung tissue due to surgery [3]-[5].

Pulmonary function test is analyzed using a spirometer. Basically, spirometry helps to study the dynamics of lungs in terms of lung volume and air flow obstructions. It also distinguishes the respiratory disease from cardiac disease [4]. Measurements such as forced expiration volume in one second, forced inspiration volume in one second and forced vital capacity are correlated to restrictive ventilatory defects, obstructive ventilatory defects, and respiratory muscle weakness. These measurements indicate various diseases such as asthma, COPD and, in turn, help to take care of the respiratory health. For this, the dynamics of lungs or volumes $\mathrm{FEV}_{1}, \mathrm{FIV}_{1}$ and maximum flows of any individual need to be compared with reference volumes obtained from normal population and accordingly patient's condition will be classified as normal and abnormal. By suitable choice of neural network architecture, classification of patients can be realized [3]-[6].

Neural networks have been a natural choice as trainable pattern classifiers because of their capability to approximate functions and to generalize [7]. Artificial Neural Networks (ANN) are powerful computational systems consisting of many simple processing elements connected together to perform tasks analogously to biological brains. They are massively parallel, which makes them efficient, robust, fault tolerant and noise independent. They can learn from training data and generalize them to new situations. The learning process of the ANN is similar to the learning function of the brain. During training, samples are presented to the input layer that yields changes of the activation state of output processing elements [7]. The calculated output value is compared to the required value which is also given in the training set. Depending upon the difference between the required and calculated output values, the network adjusts synaptic weights 
whose distribution constitutes the basis of the problem-solving algorithm. Neural networks receive extensive application in biomedical systems and have wide application in cardiology, gastroenterology, pulmonology, oncology, neurology, brain function, ophthalmology and radiology, [7] - [10].

The objective of this work is to compare the performance of two different neural network methods used for the classification of pulmonary function.

\section{METHODOLOGY}

For the present study 150 (50 Normal, 25 restrictive, 25 obstructive and 50 validation) adult volunteers were considered. The age, gender and race were identified before the test. The portable Micro lab spirometer was used for the pulmonary function test and a gold standard digital volume transducer was used for data acquisition. These transducers have already been used for precise flow volume measurements with high accuracy and stability. During the recording, the subject inhaled to total lung capacity and then exhaled as hard and completely as possible. The tracings are called flowvolume curves and are very effective in identifying normal, obstructive and restrictive ventilatory states. The acceptability and reproducibility criteria were adopted as per the recommendation of the American Thoracic Society (ATS) during measurement [3].

Forced vital capacity (FVC), forced expiratory volume in 1 second $\left(\mathrm{FEV}_{1}\right)$, ratio of $\mathrm{FVC}$ to $\mathrm{FEV}_{1}\left(\mathrm{FEV}_{1} \%\right)$, peak expiratory flow (PEF) and forced expiratory flow at $75 \%$ $\left(\mathrm{FEF}_{75} \%\right)$ were the parameters obtained through spirometric measurements and were used for the study. FVC is the volume change of the lung between a full inspiration to total lung capacity and is performed during forceful exhalation. $\mathrm{FEV}_{1}$ is the forced expiration volume in one second which is a vital parameter in pulmonary function test. Peak expiratory flow (PEF) is a measure of how fast and hard a subject can exhale while breathing out and is used to evaluate the condition of the airways in cases such as asthma and related breathing disorders. Forced expiratory flow at $75 \%\left(\mathrm{FEF}_{75} \%\right)$ is the forced flow obtained at $75 \%$ of the forced vital capacity.

The Feed forward neural network architecture with one hidden layer operating on log sigmoid transfer function has been employed for the classification of normal and abnormal data. This network has a number of simple neuron-like processing units organized in layers [10], [11]. Each unit in a layer is connected with all the units in the previous layer. The weights on these connections encode the knowledge of a network. The data enters at the input and passes through the network, layer by layer, until it arrives at the output [11]. The parameters of a network were adjusted by training the network on a set of reference data, called training set, and this training was supervised by a set of lung function labels. The training of the network was performed under back propagation of the error. The trained networks were then be used to predict labels of the new data.

Radial basis function (RBF) neural networks are also based on supervised learning. RBF networks are good at modelling nonlinear data and can be trained in one stage rather than using an iterative process as in Multilayer Layer Perceptron and also learn the given application quickly. A radial basis function network, a highly versatile and easily implementable classifier was chosen to facilitate the selection of decisive features. Radial basis function networks train rapidly (usually orders of magnitude faster than Back Propagation Network - BPN). Figure 1 shows the architectural representation of feed forward neural network [11].

The radial basis function neural network (RBFNN) is a multilayer feed forward neural network consisting of an input layer of source nodes, a layer of non linear hidden units that operate as kernel nodes and an output layer of linear weights. In response to an input vector, the outputs of the hidden layer are linearly combined to form the network response that is processed with a desired response to the output layer. The weights are trained in a supervised fashion using an appropriate linear method [11].

A radially symmetric Gaussian radial basis function was adopted as the activation function for the hidden nodes. The response of RBFNN is related to the distance between the input and the centroid associated with the basis function. The performance of the two neural network algorithms was then analyzed. The ANN used in this work were implemented using MATLAB 7 neural network toolbox.

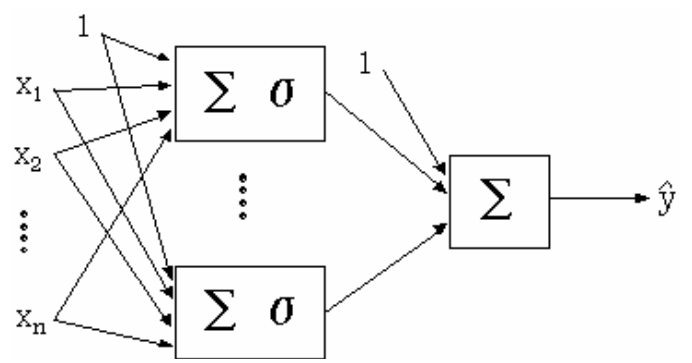

Fig.1 Architectural representation of feed forward neural network

The performance of the neural networks was estimated using False positive (FP), False Negative (FN), True Positive (TP) and True Negative (TN) values [12], [13]. Classification of a normal data as abnormal is considered as FP and classification of abnormal data as normal is considered FN. TP and $\mathrm{TN}$ are the cases where the abnormal is classified as abnormal and normal classified as normal respectively. The accuracy, sensitivity, specificity and adjusted accuracy were estimated using the following relation:

$$
\begin{aligned}
& \text { Accuracy } \quad=(\mathrm{TP}+\mathrm{TN}) /(\mathrm{TP}+\mathrm{FP}+\mathrm{TN}+\mathrm{FN}) \\
& \text { Sensitivity } \quad=\mathrm{TP} /(\mathrm{TP}+\mathrm{FN}) \\
& \text { Specificity } \quad=\mathrm{TN} /(\mathrm{TN}+\mathrm{FP}) \\
& \text { False Positive Rate }=\text { FP } /(\mathrm{TN}+\mathrm{FP}) \\
& \text { Positive Predictive Value }=\mathrm{TP} /(\mathrm{TP}+\mathrm{FP}) \\
& \text { Negative Predictive Value }=\mathrm{TN} /(\mathrm{TN}+\mathrm{FN}) \\
& \text { Adjusted accuracy }=(\text { sensitivity }+ \text { specificity }) / 2 \text {. }
\end{aligned}
$$

Accuracy is the representation of classifier performance in global sense. Sensitivity and specificity are the proportions of 
abnormal data classified as abnormal, normal data classified as normal respectively. The adjusted accuracy is a measure that accounts for unbalanced sample data of normal and abnormal events. The adjusted accuracy combines sensitivity and specificity into a single measurable value [12], [13].

\section{RESULTS AND DISCUSSIONS}

The typical response of a spirometer, which depicts variation of airflow with lung volume for a normal subject, is shown in Figure 2. The normal flow - volume curve has a distinct follow through of the inspiratory and expiratory maneuvers. In abnormal lung condition, fibrotic tissue increases the elastic recoil of the lung and this increases the airflow at a given lung volume and hence the follow through is altered. Due to this condition the peak expiratory flow is higher than the predicted value as it is evident from Figure 3. The peak expiratory flow is also narrowed due to reduction in vital capacity.

The statistical analysis of the input to the neural networks is performed and shown in Table I. The table shows the present, predicted and percentage predicted values of the input data taken for training the neural network. The mean values of the spirometer parameters for normal subjects are distinctly higher than those of the abnormal case. The standard deviation and the standard error also show significant changes. These spirometer values are given into the neural networks for training purposes and also for validation. It is observed that the sum square error was minimum (data not presented) for the considered training sets with four hidden neurons for back propagation neural networks. The performances (Table II) of the network are then calculated by giving the test data. The same work is then performed using radial basis function neural network and its performance is calculated. Table III shows the comparison of the performance of back propagation and radial basis function neural networks. It is clearly seen that radial basis function network has better accuracy when compared with the back propagation neural network. The performance of the radial basis function network was further assessed by comparing the mean and standard deviation of normal and abnormal subjects. It could be concluded that the radial basis function neural network has better accuracy and the network is efficient for the purpose for which it was trained.

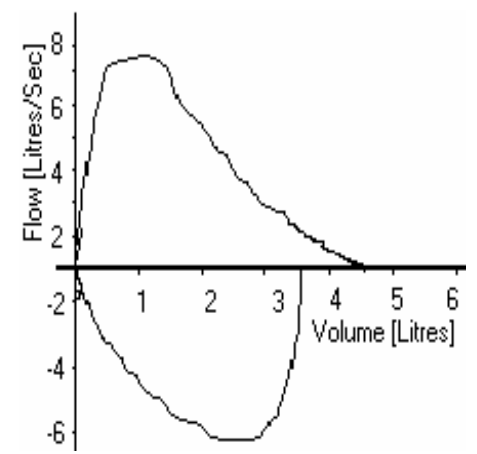

Fig.2 Variation of Flow - Volume of normal subject

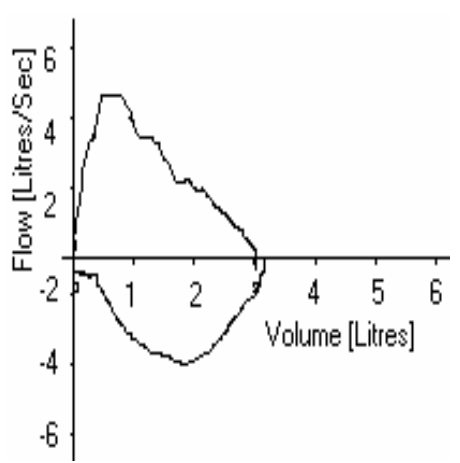

Fig.3 Variation of Flow - Volume of restrictive subject

Table I. Statistical analyses of significant parameters.

\begin{tabular}{|c|c|c|c|c|c|}
\hline \multirow{2}{*}{ Inputs } & \multirow{2}{*}{ Parameter } & \multicolumn{2}{|c|}{ Normal (50) } & \multicolumn{2}{|c|}{ Abnormal (50) } \\
\hline & & Mean \pm S.D & S.E & $\begin{array}{l}\text { Mean } \pm \\
\text { S.D }\end{array}$ & S.E \\
\hline 1 & FVC & $3.30 \pm 0.60$ & 0.07 & $\begin{array}{l}2.04 \pm \\
0.82\end{array}$ & 0.10 \\
\hline 2 & Pred $^{*}$ & $3.53 \pm 0.47$ & 0.05 & $\begin{array}{l}2.58 \pm \\
0.74\end{array}$ & 0.09 \\
\hline 3 & \% Pred ${ }^{* *}$ & $\begin{array}{l}97.89 \pm \\
16.69\end{array}$ & 2.07 & $\begin{array}{l}71.83 \pm \\
23.25\end{array}$ & 3.00 \\
\hline 4 & $\mathrm{FEV}_{1}$ & $2.75 \pm 0.52$ & 0.06 & $\begin{array}{l}1.50 \pm \\
0.56 \\
\end{array}$ & 0.07 \\
\hline 5 & Pred & $2.83 \pm 0.46$ & 0.05 & $\begin{array}{l}2.10 \pm \\
0.66\end{array}$ & 0.08 \\
\hline 6 & \% Pred & $108 \pm 21.46$ & 2.66 & $\begin{array}{l}62.52 \pm \\
18.38\end{array}$ & 2.37 \\
\hline 7 & FEV $_{1} \%$ & $83.97 \pm 8.18$ & 1.01 & $\begin{array}{l}76.42 \pm \\
19.19\end{array}$ & 2.47 \\
\hline 8 & Pred & $79.18 \pm 6.88$ & 0.85 & $\begin{array}{l}81.03 \pm \\
12.01 \\
\end{array}$ & 1.55 \\
\hline 9 & \% Pred & $112 \pm 11.19$ & 1.38 & $\begin{array}{l}93.18 \pm \\
27.01 \\
\end{array}$ & 3.48 \\
\hline 10 & FEF75\% & $1.32 \pm 0.99$ & 0.12 & $\begin{array}{l}0.30 \pm \\
0.47\end{array}$ & 0.06 \\
\hline 11 & Pred & $1.23 \pm 1.00$ & 0.12 & $\begin{array}{l}0.57 \pm \\
1.07 \\
\end{array}$ & 0.13 \\
\hline 12 & \% Pred & $144 \pm 126.08$ & 15.6 & $\begin{array}{l}32.16 \pm \\
65.83\end{array}$ & 8.49 \\
\hline 13 & PEF & $2.74 \pm 3.21$ & 0.39 & $\begin{array}{l}2.99 \pm \\
2.11\end{array}$ & 0.27 \\
\hline 14 & Pred & $3.67 \pm 4.26$ & 0.52 & $\begin{array}{l}5.04 \pm \\
2.71\end{array}$ & 0.35 \\
\hline 15 & \% Pred & $38.6 \pm 49.56$ & 6.14 & $\begin{array}{l}44.23 \pm \\
30.88\end{array}$ & 3.98 \\
\hline
\end{tabular}

"Predicted, ${ }^{* *}$ Percentage Predicted. 
Table II. Performance of (a) BP network (b) Radial basis network.

(a)

\begin{tabular}{cccc}
\hline \multirow{2}{*}{ BPN } & \multicolumn{3}{c}{ Actual Value } \\
\cline { 2 - 4 } & Normal & Abnormal & Total \\
\hline Prediction & $\mathrm{TP}=23$ & $\mathrm{FP}=2$ & 25 \\
\hline $\begin{array}{c}\text { False } \\
\text { Negative }\end{array}$ & $\mathrm{FN}=0$ & $\mathrm{TN}=25$ & 25 \\
\hline Total & 23 & 27 & $\mathbf{5 0}$ \\
\hline
\end{tabular}

(b)

\begin{tabular}{cccc}
\hline \multirow{2}{*}{ RBF } & \multicolumn{3}{c}{ Actual Value } \\
\cline { 2 - 4 } & Normal & Abnormal & Total \\
\hline Prediction & $\mathrm{TP}=25$ & $\mathrm{FP}=0$ & 25 \\
\hline $\begin{array}{c}\text { False } \\
\text { Negative }\end{array}$ & $\mathrm{FN}=0$ & $\mathrm{TN}=25$ & 25 \\
\hline Total & 25 & 25 & $\mathbf{5 0}$ \\
\hline
\end{tabular}

Table III. Comparison of RBF and BPN

\begin{tabular}{|l|l|l|}
\hline \multicolumn{1}{|c|}{ Indices } & BPN & RBF \\
\hline Accuracy & $96 \%$ & $100 \%$ \\
\hline $\begin{array}{l}\text { Sensitivity } \\
\text { True positive rate }\end{array}$ & $100 \%$ & $100 \%$ \\
\hline Specificity & $92.59 \%$ & $100 \%$ \\
\hline $\begin{array}{l}\text { False Positive Rate } \\
\text { (1 - Specificity) }\end{array}$ & $7.41 \%$ & $0 \%$ \\
\hline Positive Predictive Value & $92 \%$ & $100 \%$ \\
\hline Negative Predictive Value & $100 \%$ & $100 \%$ \\
\hline
\end{tabular}

\section{CONCLUSION}

Lung function analysis plays an important role for the diagnosis, prognosis, mass screening of respiratory disorders and the spirometric investigations remain central in clinical practice [14]. It has been shown that $50 \%$ of the spirometric results were unacceptable due to failure to complete the test [14]-[17] as these investigations depend on the ability of the investigated subject to complete the test and on the skills and approaches of the investigator. Hence attempts are being made to utilize the artificial intelligence methods to classify the pulmonary function data [16].

In this work, spirometer data are classified into normal and abnormal cases, using artificial neural networks in detail. The performance comparisons of two neural network algorithms are assessed. A solution for the classification of the spirometer data using neural network has been generated for unknown cases and are consulted for validation. The conclusions are drawn after rigorous experimentation on best architecture, number of hidden neurons required and goal.
It is observed that radial basis function networks have better accuracy when compared to back propagation neural networks. The value of specificity shows that radial basis function network classifies abnormal data more accurately than back propagation network. The positive predictive value suggests that the classification of spirometric data into normal is higher in the radial basis function than that of the back propagation network. The negative predictive value indicates that the back propagation network diagnoses the abnormal data more correctly than the normal data, where as both the normal and abnormal data are correctly diagnosed in the radial basis function network. However, the accuracy of the back propagation network could be further enhanced by including more spirometric parameters and a large database for training.

It appears that ANN could be a valuable alternative to statistical methods. The proposed methodology could be effective for mass screening and surveying of respiratory function gross abnormalities at primary care settings.

\section{ACKNOWLEDGEMENT}

The authors would like to thank Dr. R. Sridharan for his help in clinical data collection.

\section{REFERENCES}

[1] Pierce, R. (2005). Spirometer: an essential clinical measurement. Aust. Fam. Physician 34 (7), 535-539.

[2] Oud, M. and Maarsingh, E.J.W. (2004). Spirometer and forced oscillatory assisted optimal frequency band determination for the computerized analysis of tracheal lung sounds in asthma. Physiol. Meas. 25, 595-606.

[3] Miller, M.R., Hankinson, J., Brusasco, V., Brugos, F., et al. (2005). Standardisation of spirometry. Eur. Respir. J. 26 (2), 319-338.

[4] Arora, V.K. and Raghu, S. (2000). Flow volume curves: clinical significance. Lung India 14 (4), 169 - 171.

[5] Mark, D. S., Micharl, W. K., Mitchell, L. D., James, R.Y. and Michael, R.K. (2006). Classifying severity of cystic fibrosis lung disease using longitudinal pulmonary function data. Am. J. Respir. Crit. Care Med. 174 (7), 780-786.

[6] Kornel, P., Bela, M., Rainer, S., Zalan, D., Zsolt, T. and Janos, F. (1998). Application of neural network in medicine. Diag. Med. Tech. 4 (3), 538-546.

[7] Gaetano, P. (2004). Artificial neural network in the assessment of respiratory mechanism. Unpublished doctoral dissertation, Faculty of Medicine, University of Upsaliensis, Uppsala.

[8] Botis, T. and Halkiotis, S. (2003). Neural networks for the prediction of spirometric reference values. Med. Inform. Internet Med. 28 (4), 299-309.

[9] Juroszek, B. (2005). The Influence of gas parameters on the result of spirometric test. Meas. Sci. Rev. 5 (2), 25-28.

[10] Ofer, B., Shimon, A., Alexander, G. and Israel, B. (2004). Model-Based prediction of expiratory resistance index in patients with asthma. J. Clinic Monit. Comput. 18 (4), 241-245. 
[11] Joon, L., Stefanie, B., Mike, J.C., David, J.K., Glenn, B. and Tom, C. (2006). A radial basis classifier for the automatic detection of aspiration in children. $J$. NeuroEng. Rehabil. 3, 1-17.

[12] Abboud, S., Barnea, O., Guber, A., Narkiss, N. and Bruderman, I. (1995). Maximum expiratory flow-volume curve: mathematical model and experimental results. Med. Eng. Phys. 17 (5), 332-336.

[13] Gaetano, P., Marieann, H., Christian, R., Rocco, G. and Tommaso, F. (2001). Assessment of respiratory mechanism by artificial neural network: an exploratory study. J. Appl. Physiol. 90 (5), 1817-1824.

[14] Jouni, H., Jaakko, K., Tuija, P. and Markku, M.N. (1999). Prevalence of asthma, aspirin intolerance, nasal polyposis and chronic obstructive pulmonary disease in a population-based study. Int. J. Epidemiol. 28 (4), 717722.

[15] Mahesh, V. and Ramakrishnan, S. (2007). Detection of obstructive respiratory abnormality using flow-volume spirometry and radial basis function neural networks. $J$. Med. Syst. 31 (6), 461-465.

[16] Mahesh, V. and Ramakrishnan, S. (2007). Neural network based classification of normal and abnormal pulmonary function using spirometric measurements. $J$. Mech. Med. Bio. 7 (2), 151-161.

[17] Ulmer, W.T. (2003). Lung Function-Clinical importance, problems, and new results. J. Physiol Pharmacol. 54 (1), 11-13. 